\title{
Treatment Approach for Oral Candidiasis: Two Case Reports
}

\author{
Gaye Keser 1 , Filiz Namdar Pekiner \\ Department of Oral Diagnosis and Maxillofacial Radiology, Marmara University, Faculty of Dentistry, Istanbul, Turkey. \\ Correspondence Author: Gaye Keser \\ E-mail: gaye.sezgin@marmara.edu.tr \\ Received: 18.01.2021 Accepted: 04.04.2021
}

\begin{abstract}
Candida infections are acute and / or chronic infections of the skin, mucosa, internal organs and systems which may be seen at any age. The purpose of this study is to present diagnostic and therapeutic approach for patients who applied to our clinic with the diagnosis of pseudomembranous candidiasis. Both patients who referred to our clinic were using inhaler due to Chronic Obstructive Pulmonary Disease (COPD). Intraoral examination revealed pseudomembranous candidiasis localized on both the border of soft palate and through pharynx of a 60 year-old female patient and on the midline of hard palate of a 70-year-old male patient. Cultures were taken for mycological evaluation of the patients after clinical examination. Antifungal treatment was administered to both patients for 10 days. Healing was observed as a result of the treatment. No recurrence was observed at monthly follow-up assessments performed for patients. In cases of oral candidiasis, antifungal agents should be used locally in the form of suspension or pomade, evaluation of mycological culture should be performed to support clinical diagnosis and the etiological factors that may cause the disease should be studied.
\end{abstract}

Keywords: oral candidiasis, fungal infection, treatment approach

\section{INTRODUCTION}

The most frequent fungal infection is candidiasis, and it is caused by Candida albicans, a fungus present in $20-50 \%$ of healthy individuals. Other strains (Candida tropicalis, Candida parapsilosis, Candida glabrata, Candida krusei, Candida guilliermondii) are rarely responsible for candidiasis (1). In healthy individuals, without any local predisposing factor, $C$. albicans causes no clinical manifestations. Based on age and predisposing variables, multiple incidences were found $(2,3)$. Predisposing factors for candidiasis include local ones, such as xerostomia, poor oral hygiene, chronic mucosal trauma, use of local antibiotics, chronic use of inhalational and topical steroids, radiotherapy to the head area, and systemic ones, such as iron deficiency anemia, diabetes mellitus, primary immunodeficiency, HIV infection and AIDS, leukemia and other malignancies, neutropenia, use of steroids and immunosuppressive medication, broad-spectrum antibiotics, hypoparathyroidism, cortical adrenal insufficiency, and other endocrine diseases $(1,3,4)$.

Oral candidiasis is prevalent and underdiagnosed in the elderly, especially in those wearing dentures, and can be avoided in certain instances with a proper mouth care regimen $(5,6)$. A wide variety of clinical symptoms are found in oral mucosal candidiasis (3-9). The diagnosis is mainly based on the history and clinical features with further investigations required only for difficult cases. Identification and possible resolution of local or systemic causative factors are essential for the treatment of candidal infection. Nystatin and miconazole are the drugs of choice for topical use. Triazoles (e.g., fluconazole, itraconazole) are useful systemic agents. The dosage and duration of treatment largely depends on the type of candidiasis and the presence of predisposing factors $(7,8)$.

\section{CASE PRESENTATION}

\subsection{Case I}

A 60-year-old female patient was referred to the Department of Oral Diagnosis and Radiology Clinic of the Marmara University Faculty of Dentistry by a clinician who observed unhealed oral mucosal lesions on hard and soft palate for one and a half month. A medical history revealed that she had Chronic Obstructive Pulmonary Disease (COPD) and she was treated with bronchodilator (inhaler) as a reliever. On intraoral examination, whitish-yellow, slightly elevated spots were observed on her hard palate and soft palatal region (Fig. 1a, b). On scratching, lesions became scrapable and left diffuse erythematous patches. Smear made from lesion scraping was sent for cytological analysis to validate the existence of candidate hyphae (Fig. 2). Final diagnosis of drug-induced 
pseudomembranous candidiasis was performed on the basis of history, clinical appearance and cytological report. For around 10 days, the patient was recommended to use Nystatin suspension about 4-5 times a day. Within 15 days, the patient was witnessed a serious remission of the lesions (Fig. 3).

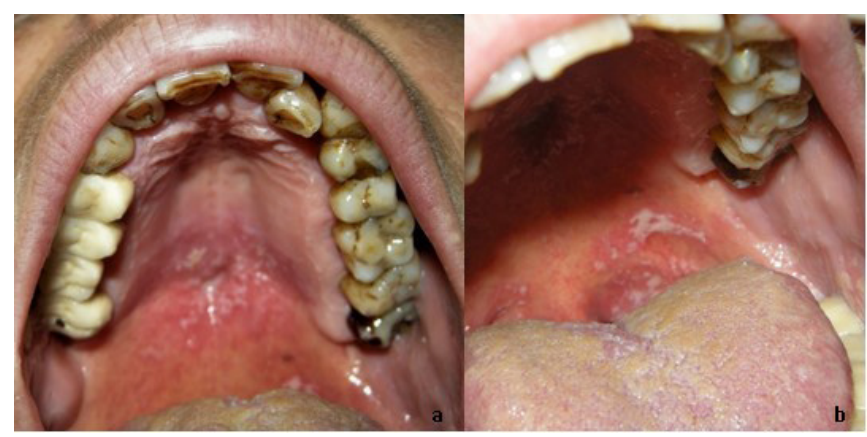

Figure 1. Whitish-yellow, slightly elevated scrapable spots underlying erythematous surface $(a, b)$ on the midline of hard palate.

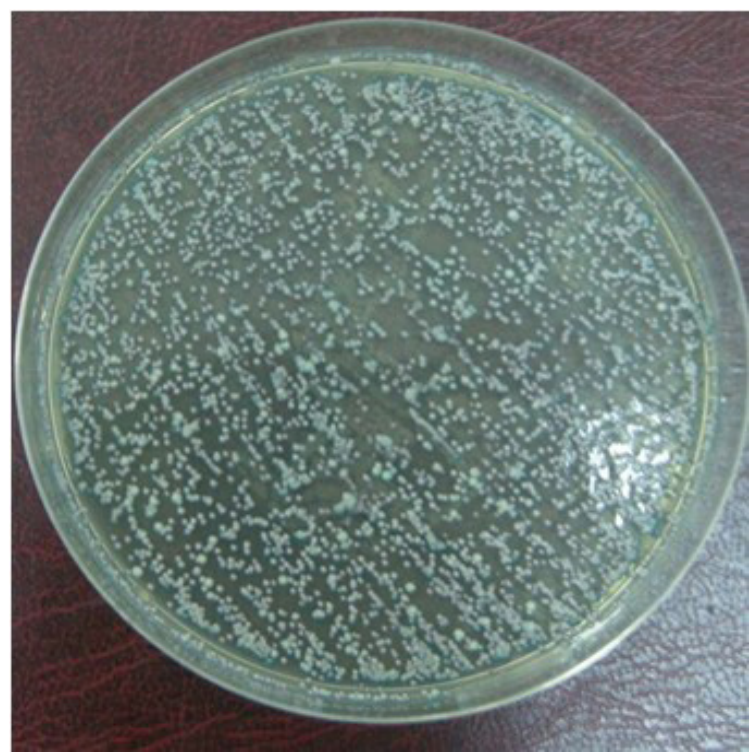

Figure 2. Culture of samples obtained from first case showing candidal colonies (C.albicans)

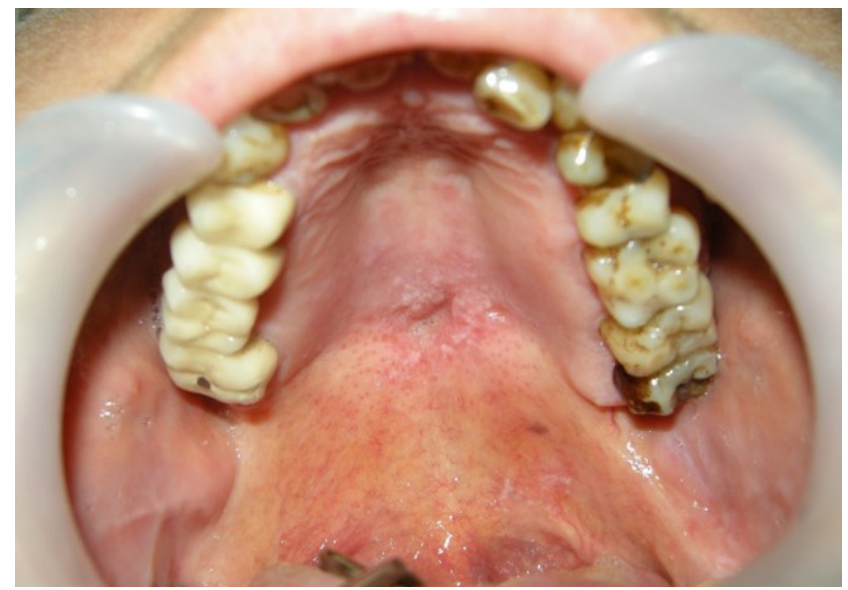

Figure 3. Remission of the lesions after antifungal therapy

\subsection{Case II}

A 70 - year-old male patient was referred to the Department of Oral Diagnosis and Radiology Clinic of the Marmara University Faculty of Dentistry by a clinician who observed unhealed oral mucosal lesions on hard and soft palate for one and a half month. A medical record showed he was hypertensive and had COPD. The patient was treated with bronchodilator (inhaler) as a reliever. On intraoral examination, whitish-yellow, slightly elevated spots were observed on the midline of hard palate. (Fig. 4). The lesions become scrapable upon scraping and left dispersed erythematous patches. Smear from lesion scraping was submitted to verify the presence of candidate hyphaee for cytological examination (Fig. 5). On the basis of history, clinical presentation and cytological findings, the final diagnosis of drug-induced pseudomembranous candidiasis was carried out. The patient was also advised to use Nystatin suspension around 4-5 times per day for about one week. After the procedure, the patient was tested and displayed a serious remission of the lesions (Fig. 6).

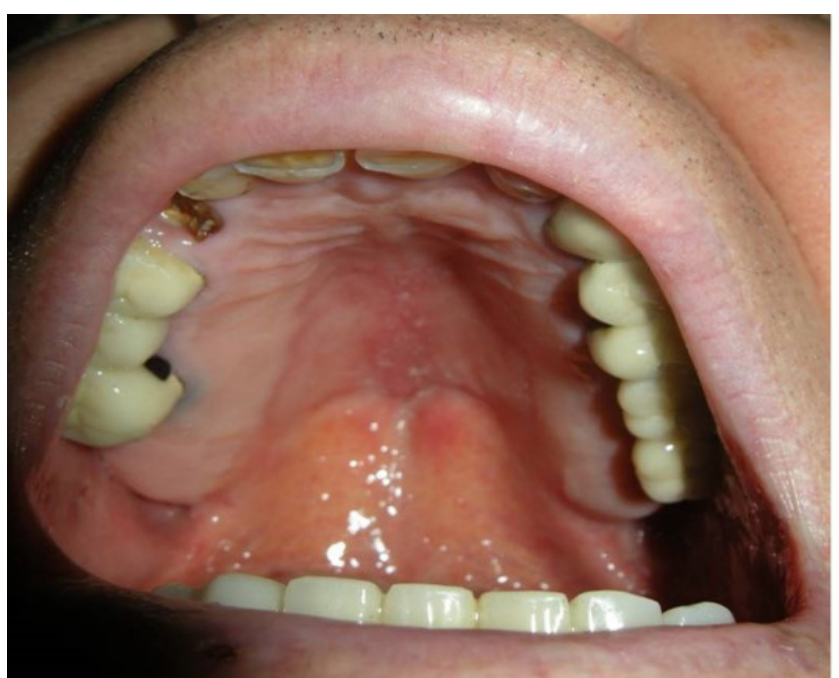

Figure 4. Whitish-yellow, slightly elevated scrapable spots underlying erythematous surface on the midline of hard palate.

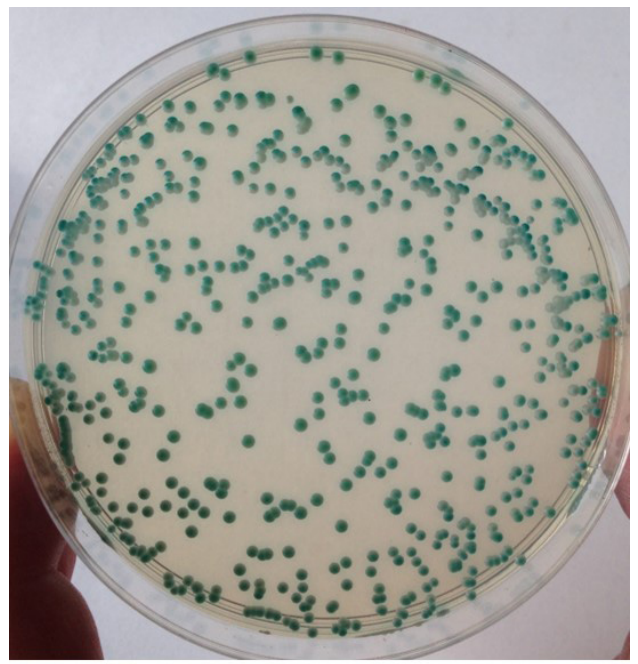

Figure 5. Culture of samples obtained from second case showing candidal colonies (C.albicans). 


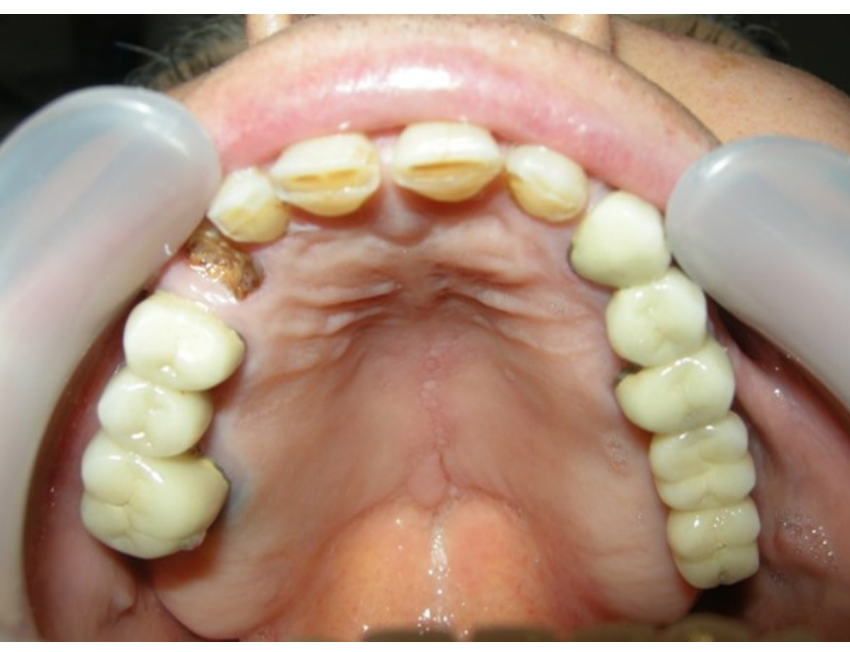

Figure 6. Remission of the lesions after antifungal therapy.

\section{DISCUSSION}

In patients with systemically immunocompromised diseases, fungal human infections caused by distinct Candida species vary from such inconsequential conditions such as oral or genital candidiasis to super-infections $(9,10)$. Levels of frequency of $C$. albicans have been recorded as being between $45-65 \%$ and $30-45 \%$, respectively, in the oral cavity of neonates, healthy children and healthy adults. In comparison, the prevalence figures are 50-65\% of persons wearing removable dentures, $65-88 \%$ of those in acute and long-term care hospitals, $90 \%$ of chemotherapy patients with acute leukemia, and $95 \%$ of HIV patients.

Physiological causes such as old age, childbirth and pregnancy with altered immunity, local trauma, poor dental hygiene, malnutrition, use of broad-spectrum antibiotics, corticosteroids and inhalers, immune deficiencies such as HIV infection, endocrine disorders, malignancies such as leukemia, agranulocytosis, hyposalivation due to autoimmune diseases or head and neck radiation are certain reasons that predispose the host to oral candidiasis (11).

Oral candidiasis treatment should be in the direction for identification and correction of factors that may affect or contribute to the occurrence of oral candidiasis together with a detailed history (12). Any deficiency conditions (iron, folate, vitamin B12 and C) as well as diabetes mellitus or any immunodeficiency state should be investigated. Any pharmacological agent that may contribute to the occurrence of oral candidiasis should be determined and if possible, it should be used instead of an alternative drug. Salivary gland functions should be checked and, if necessary, saliva flow should be measured (13). Adequacy of prostheses in patients with denture stomatitis must also be considered. Oral and prosthetic hygiene should be evaluated and corrected. In addition, patients should be advised not to wear the prosthesis continuously day and night (14). If it is not possible to correct the underlying factors or, if not indicated, pharmacological treatment is required.
The mechanism of action of antifungal agents is to change the metabolism of RNA and DNA or to accumulate peroxide in the cell that is harmful to the fungal cell. Topical polyene or antifungal agents of azole are often effective. In most cases, patients are successful with Nystatin oral suspension $(100,000$ units/mL-1 mL topically) or nystatin pastilles (100,000 IU) four times a day for 7-14 days. Amphotericin tablets $(10 \mathrm{mg})$ or suspension tablets (100 mg / mL) four times a day for 14-21 days after meals are also realistic. In addition, $2 \%$ Miconazole gel, approximately $2.5 \mathrm{~mL}$ topically, can be used four times a day after meals for 14-21 days. Miconazole is contraindicated in patients taking warfarin as it potentiates its effects $(14,15)$.

In these reported cases, patients were also analyzed for potential structural factors alongside a proper history and clinical examination. Upon delivery of the final diagnosis, the appropriate therapy was selected by adding the antifungal agent nystatin. Against a large spectrum of yeasts and yeastlike fungi, nystatin is both fungistatic and fungicidal in vitro (9-11,14). In repeated subculture, Candida albicans shows no substantial resistance to nystatin in vitro in increasing nystatin levels and therapy has been adequately successful in healing lesions. When patients were re-evaluated at the follow-up visit, complete remission of the lesions was found.

\section{CONCLUSION}

The key duties of every dental specialist are diagnosing oral candida lesions together with effective treatment steps. In order to diagnose this clinical issue, proper documentation of medical history is necessary. Where feasible, predisposing factors should be handled or removed. In conclusion, two reports of oral candidiasis triggered by an inhaler have been reported. These forms of reactions are normal in patients receiving an inhaler, so we highly suggest that diagnosis should be carried out immediately. Timely treatments should be carried out and pursued in order to minimize this adverse effect among patients receiving inhaler therapy. Nystatin therapy can be effective in cases of inhaler-induced oral candidiasis.

\section{ACKNOWLEDGMENT}

We thank Prof. Tanju Kadir for assistance with laboratory isolation and identification of candida species. This study is presented as a poster at the 1st International Dental Symposium of Marmara University Faculty of Dentistry, Istanbul, Turkey, May 4-5, 2018.

\section{REFERENCES}

[1] Laskaris G. Fungal infections. In: Color Atlas of Oral Diseases Springer. Thieme, 4th ed. Stuttgart; 2005.p.238-242.

[2] Liu X, Hua H. Oral manifestation of chronic mucocutaneous candidiasis: seven case reports. J Oral Pathol Med 2007; 36:528-532.

[3] Motta-Silva AC, Aleva NA, Chavasco JK, Armond MC, França $J P$, Pereira LJ. Erythematous oral candidiasis in patients with 
controlled type II diabetes mellitus and complete dentures. Mycopathologia 2010; 169:215-223.

[4] Muzyka BC, Epifanio RN. Update on oral fungal infections. Dent Clin North Am 2013; 57:561-581.

[5] Limbhore MV, Ramanojam S, Rathi P, Sane V, Mevawala A. Oral candidiasis:s: An overview and case report. Era J Med Res 2019; 6: 1-7.

[6] Singh A, Verma R, Murari A, Agrawal A. Oral candidiasis: An overview. J Oral Maxillofac Pathol. 2014;18: S81-S85.

[7] Lyu X, Zhao C, Yan ZM, Hua H. Efficacy of nystatin for the treatment of oral candidiasis: a systematic review and metaanalysis. Drug Des Devel Ther 2016;10: 1161-1171.

[8] Millsop JW, Fazel N. Oral candidiasis. Clin Dermatol 2016 JulAug;34:487-494.

[9] Patil A, Susmitha HR, Basappa S, Mahesh MS. Drug-induced oral candidiasis: A case report. . IJSS Case Reports \& Reviews 2016;2:1-4.
[10] Imron Nasution A. Virulence factor and pathogenicity of Candida albicans in oral candidiasis. G Patil S, ed. WJOUD 2013;4:267-271.

[11] Williams D, Lewis M. Pathogenesis and treatment of oral candidosis. J Oral Microbiol 2011;3:10.3402/jom.v3i0.5771.

[12] Borahan MO, Keser G. Ağız Mukozasında Mantar Enfeksiyonları. Illgüy D, editör. Ağız Hastalıkları. Ankara: Türkiye Klinikleri; 2018.p.71-76 (Turkish).

[13] Giannini PJ, Shetty KV. Diagnosis and management of oral candidiasis. Otolaryngol Clin North Am 2011;44:231-240.

[14] Krishnan PA. Fungal infections of the oral mucosa. Indian J Dent Res 2012;23:650-659.

[15] Figueiral MH, Azul A, Pinto E, Fonseca PA, Branco FM, Scully C. Denture related stomatitis: identification of aetiologicaland predisposing factors-a large cohort. J Oral Rehabil 2007;34:448455.

How to cite this article: Keser G, Namdar Pekiner F. Treatment Approach for Oral Candidiasis: Two Case Reports. Clin Exp Health Sci 2021; 11: 360-363. DOI: 10.33808/clinexphealthsci.753980 